\title{
Epidemiological and genetic characterization of Clostridium butyricum cultured from neonatal cases of necrotizing enterocolitis in China
}

\author{
Yinping Dong MD¹, Ying Li MD², Di Zhang BA², Scott Nguyen $\mathrm{PhD}^{3}$, Nikunj Maheshwari $\mathrm{PhD}^{3}$, Yujie Hu MD ${ }^{1}$, Yu Cao PhD², \\ Fengqin Li PhD ${ }^{1}$ and Séamus Fanning $\mathrm{PhD}^{1,3}$ \\ ${ }^{1} \mathrm{NHC}$ Key Laboratory of Food Safety Risk Assessment, China National Center for Food Safety Risk Assessment, Beijing, PR China, ${ }^{2}$ Children's Hospital Affiliated \\ with Capital Institute of Pediatrics, Beijing, PR China and ${ }^{3}$ School of Public Health, Physiotherapy \& Sports Science, University College of Dublin, Dublin, Ireland
}

\begin{abstract}
Objective: Laboratory-based characterization and traceback of Clostridium butyricum isolates linked to outbreak cases of neonatal necrotizing enterocolitis (NEC) in a hospital in China.

Methods: In total, 37 samples were collected during the NEC outbreak. Classical bacteriological methods were applied to isolate and identify Clostridium spp. Meanwhile, 24 samples collected after an outbreak were similarly tested. All Clostridium isolates were identified to species level as either C. butyricum or C. sporogenes. These isolates were subsequently subtyped using pulsed-field gel electrophoresis (PFGE). Genomic DNA was purified from 2 representative C. butyricum isolates and sequenced to completion.

Results: Of 37 samples collected during the NEC outbreak, 17 (45.95\%) were positive for Clostridium spp. One species, C. butyricum, was cultured from 10 samples. Another species cultured from 2 other samples was identified as C. sporogenes. Both of these species were cocultured from 5 samples. Pulsotyping showed that the 15 C. butyricum and the 7 C. sporogenes isolates produced indistinguishable DNA profiles. No NEC cases were reported after disinfection following the outbreak, and all samples collected after the outbreak were negative for Clostridium spp. Whole-genome sequencing (WGS) indicated that sialidase, hemolysin, and enterotoxin virulence factors were located on the chromosomes of 2 C. butyricum isolates.
\end{abstract}

Conclusions: The outbreak of NEC was epidemiologically linked to C. butyricum contamination within the hospital. This is the first report of an NEC outbreak associated with C. butyricum infection in China.

(Received 29 May 2019; accepted 15 September 2019; electronically published 16 June 2020)

Necrotizing enterocolitis (NEC) is a severe and life-threatening, acquired gastrointestinal disorder among preterm neonates, especially infants of very low birth weight $(<1,500 \mathrm{~g}) \cdot{ }^{1-3} \mathrm{NEC}$ is characterized by abdominal distension, blood in the stool, portal venous gas, pneumatosis intestinalis, and pneumoperitoneum, with reported mortality ranging from $20 \%$ to $50 \%{ }^{1}$ Although NEC pathophysiology remains unclear, prematurity, enteral feeding strategies, gut bacterial colonization, and inappropriate proinflammatory response are major factors implicated in NEC development. ${ }^{4}$ Several causative microorganisms including viruses, gram-negative bacilli, and Clostridium spp have been proposed. ${ }^{5}$ No etiologic agent has been definitively established; nonetheless, the most commonly implicated bacteria include members of the Clostridium genus, especially C. butyricum. ${ }^{6}$ Cytotoxic C. butyricum isolates obtained from a larger population of preterm neonates with NEC strengthen the

Author for correspondence: Fengqin Li, E-mail: lifengqin@cfsa.net.cn. Or Séamus Fanning, E-mail: sfanning@ucd.ie

Cite this article: Dong Y, et al. (2020). Epidemiological and genetic characterization of Clostridium butyricum cultured from neonatal cases of necrotizing enterocolitis in China. Infection Control \& Hospital Epidemiology, 41: 900-907, https://doi.org/10.1017/ ice.2019.289 etiology of C. butyricum in cases of NEC, independent of intrinsic factors. ${ }^{7,8}$ NEC-like lesions produced by C. butyricum in animal models as well as in Caco- 2 cell lines have also been successfully duplicated, further demonstrating the cytotoxic activity of this bacterium..$^{9-12}$ Genome sequencing of the isolates cultured from specimens linked to NEC patients allowed the identification of genes encoding polypeptides that are highly similar to hemolysins shared by Brachyspira hyodysenteriae. ${ }^{13}$

In this study, we investigated an outbreak of NEC in Shandong Province, China. We carried out the laboratory-based epidemiological traceability and genetic characterization of C. butyricum isolated from specimens linked to NEC outbreak to explore the potential role of C. butyricum in the pathogenesis of NEC.

\section{Materials and methods}

\section{Ethics statement}

Experiments related to infant fecal specimens were performed in accordance with the written informed consent from each patient's

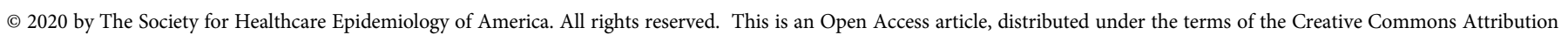
licence (http://creativecommons.org/licenses/by/4.0/), which permits unrestricted re-use, distribution, and reproduction in any medium, provided the original work is properly cited. 
guardian. The study protocol was approved by the Ethics Committee of China National Center for Food Safety Risk Assessment.

\section{Case overview}

From October through November 2016, an outbreak of NEC occurred in a maternal and child health-care hospital in Shandong Province, China. Infants who presented with bloody stools, abdominal distension with or without drowsiness, apnea, hypotonia, and/or pneumatosis intestinalis were diagnosed as NEC cases. In total, 15 cases were recorded within 1 month in the neonatal intensive care unit (NICU), and all patients were neonates. Among them, 5 were premature neonates born in this hospital and were subsequently diagnosed with NEC 4-6 days after birth. The other 10 NEC cases were neonates delivered at full term in other hospitals who had been admitted to the onset hospital (where the NEC index was reported) as a result of unrelated complications. All 10 of these neonates were discharged from the onset hospital having made a full recovery from their primary disease, after which they were all subsequently readmitted to the onset hospital. All 15 NEC cases had the same clinical symptoms and signs, including bloody stools and abdominal distension without fever and infectious sepsis. These infants were fed with either breast milk or infant formula powder prior to their NEC diagnosis.

\section{Sample collection}

In total, 61 samples, including 37 taken from sources linked to the NEC outbreak and 24 others (including 1 breast milk and 23 environmental samples) after disinfectant interventions following the outbreak, respectively, were collected for microbiological analysis. Among these 15 NEC patients, 11 had been discharged originally from the hospital at the time of sample collection. Another 2 patients who remained hospitalized at the index hospital did not produce sufficient stool samples for testing due to receiving fasting and water-free treatment strategies after being diagnosed as NEC. Only 2 stool samples were successfully collected from 2 NEC case patients (cases A and B). In addition, 8 hand swabs were obtained from 8 nurses responsible for taking care of these children in the ward at the time of sampling. All of these samples were collected by swabbing a $10-\mathrm{cm} \times 10-\mathrm{cm}$ surface area of these objects with surface samplers (UXL100, 3M, Cottage Grove, MN) before depositing them into tubes containing buffered peptone water (BPW). Further details of the sampling methods are included in Table 1.

\section{Laboratory analysis}

Isolation, identification of Clostridium spp. Clostridium was isolated in accordance with the US Food and Drug Administration's Bacteriological Analytical Manual (Chapter 17: Clostridium botulinum $).{ }^{14}$ Presumptive colonies were selected for gram staining, microscopic examination, API 20A and VITEK2 (bioMérieux, Marcy l'Etoile, France) biochemical testing, and 16S rRNA gene sequencing in accordance with the recommended procedures.

PFGE molecular subtyping. Presumptive Clostridium spp cultured from the samples described above were purified, and PFGE subtyping was performed according to the previously published method. ${ }^{15}$

Whole-genomic sequencing. Two C. butyricum isolates cultured from samples of the patient's stool and a swab taken from the inner wall of NEC-infant's incubator coded F1-b and F5-b, respectively, were recovered, followed by genomic DNA purification. Wholegenome sequencing was performed using the Pacific Biosciences
RS II platform (SMRT, Pacific Biosciences, Menlo Park, CA). De novo assembly of the reads was carried out using Hierarchical Genome Assembly Process (HGAP) version 3.0 software module.

\section{Bioinformatical analysis}

The 2 complete $C$. butyricum genomes sequenced in this study were compared with a reference C. butyricum KNU-L09 genome (accession no. NZ_CP013252). A bioinformatics framework (seq-seq-pan), designed to construct a pangenome from a set of aligned genomes, was used to construct a $C$. butyricum pangenome from all 3 genomes. ${ }^{16}$ A BLAST ring image generator (BRIG) was used to construct a comparative genomic representation of the 3 C. butyricum genomes against the pangenome. ${ }^{17}$ Putative prophages were identified using the PHASTER web server. ${ }^{18}$ Genomic islands and other mobile genetic elements (MGE) were manually inspected and determined by Mauve alignment of the genomes in Geneious version 8.1.6 software (Biomatters, Auckland, New Zealand). ${ }^{19}$ The virulence factor database (VFDB, http://www.mgc.ac.cn/VFs/ main.htm) was used to predict the presence of virulence factors in the genomes of the sequenced strains. Clostridium butryicum sequences were downloaded from the National Center for Biotechnology Information (NCBI) using the ncbi-genomedownload script (https://github.com/kblin/ncbi-genome-download). Parsnp from the Harvest suite was used to construct a phylogenetic tree of the C. butyricum sequences in conjunction with the 2 sequenced strains in this study. ${ }^{20}$ Parsnp was run with default options, and C. butryicum KNU-L09 was included as the reference for the phylogenetic tree; Evolview 2 was used to visualize the tree. ${ }^{21}$ Nucleotide sequences and statistics regarding 2 C. butyricum isolates were submitted to GenBank.

\section{Results}

\section{Clostridium isolation and identification}

In total, 37 samples were collected during the NEC outbreak, and 17 (45.95\%) were positive for a presumptive Clostridium. Also, 15 samples $(40.50 \%)$ were contaminated with C. butyricum, as determined by gram staining, microscopic examination, biochemical testing, and 16S rRNA gene sequencing. These positive samples included the leftover powdered infant formula (PIF) sample initially consumed by the neonates linked to the NEC cases, feces of NEC patients, swab samples taken from the hands of the attending medical staff, disposable items for neonate daily use, breast milk bags, medical equipment used in the ward (eg, infusion pump, an electrocardiogram (ECG) monitor, stethoscope, telephone, refrigerator, as well as handle and inner wall surfaces of the neonate incubator) (Table 2). Of 37 samples, 5 (13.5\%) including the feces of 2 NEC patients, swab samples taken from the inner wall of a refrigerator, the door handle of the incubator as well as disposable items for daily use were cocontaminated with both C. butyricum and C. sporogenes. Two samples (5.4\%) were positive for C. sporogenes alone (Table 2).

Clostridium butyricum cultured from NEC-related samples produced colonies were white in color, semitransparent, and had irregular contours and rhizoid colonies on Columbia blood agar (Fig. 1). When subjected to a gram staining observed under high-phase microscopy, these bacterial cells resembled a tennisracket shape in which the oval-shaped spores were greater in width than the bacterium. 
Table 1. The Information of Samples Analyzed

\begin{tabular}{|c|c|c|c|}
\hline Sampling Time & Sample Source & Sample Name & No. of Samples \\
\hline \multirow[t]{23}{*}{ During an NEC outbreak } & \multirow[t]{3}{*}{ Related to 2 NEC cases } & Stools & 2 \\
\hline & & Leftover powdered infant formula & 2 \\
\hline & & Breast milk & 2 \\
\hline & Related to a non-NEC case & Breast milk & 1 \\
\hline & \multirow[t]{12}{*}{ Ward environment } & Swabs of inner wall of non-NEC infant incubator & 4 \\
\hline & & Swab of inner wall of the breast milk bag & 1 \\
\hline & & Swab of inner wall of the NEC-infant incubator & 1 \\
\hline & & Swab of handle of the NEC-infant incubator & 1 \\
\hline & & Swab of inner wall of refrigerator & 1 \\
\hline & & Swab of milk-preparation desk & 1 \\
\hline & & Swab of treatment table & 1 \\
\hline & & Swab of ECG monitor & 1 \\
\hline & & Swab of infusion pump & 1 \\
\hline & & Swab of stethoscope & 1 \\
\hline & & Swab of public phone & 1 \\
\hline & & Swab of baffle of radiation probe & 1 \\
\hline & Medical staff & Hand swabs & 8 \\
\hline & \multirow[t]{5}{*}{ Articles for neonate daily use } & Swab of inner wall of a box for holding breast milk bag & 1 \\
\hline & & Swabs of inside of the feeding bottles & 2 \\
\hline & & Swabs of nipple of feeding bottles & 2 \\
\hline & & Swab of outside of the cap of a feeding bottle & 1 \\
\hline & & Water & 1 \\
\hline & Total & & 37 \\
\hline \multirow{19}{*}{$\begin{array}{l}\text { After interventions } \\
\text { following outbreak }\end{array}$} & Related to the non-NEC case & Breast milk & 1 \\
\hline & \multirow[t]{10}{*}{ Ward environment } & Swabs of inner wall of the case patient's incubator & 2 \\
\hline & & Swab of inner wall of refrigerator & 1 \\
\hline & & Swab of ECG monitor & 1 \\
\hline & & Swab of infusion pump & 1 \\
\hline & & Swab of baffle of radiation probe & 1 \\
\hline & & Sink & 1 \\
\hline & & Swab of inner wall of tourniquet box & 1 \\
\hline & & Swabs of public phone & 2 \\
\hline & & Swabs of computer mouse & 2 \\
\hline & & Swabs of computer keyboard & 2 \\
\hline & Medical staff & Hand swabs & 2 \\
\hline & \multirow{6}{*}{$\begin{array}{l}\text { Articles for neonate } \\
\text { daily use }\end{array}$} & Swab of inner wall of feeding bottle cabinet & 1 \\
\hline & & Swab of inner wall of a box for holding breast milk bag & 1 \\
\hline & & Swab of inside of the feeding bottle & 1 \\
\hline & & Swab of nipple of a feeding bottle & 1 \\
\hline & & Water & 1 \\
\hline & & Powdered infant formula & 2 \\
\hline & Total & & 24 \\
\hline Total & & & 61 \\
\hline
\end{tabular}


Table 2. The Results of Clostridium spp Isolation and Identification

\begin{tabular}{|c|c|c|c|c|c|c|}
\hline \multirow[b]{2}{*}{ Sample Source } & \multirow[b]{2}{*}{$\begin{array}{l}\text { Sample } \\
\text { Code }\end{array}$} & \multirow[b]{2}{*}{ Sample Name } & \multicolumn{4}{|c|}{ Identification Results } \\
\hline & & & Biochemical Test & $\begin{array}{l}\text { 16S rRNA Gene } \\
\text { Sequencing }\end{array}$ & Final & $\begin{array}{l}\text { Strain } \\
\text { Code }\end{array}$ \\
\hline \multirow[t]{6}{*}{ Samples associated with case A } & \multirow[t]{2}{*}{$\mathrm{F} 1$} & \multirow[t]{2}{*}{ Stool } & C. butyricum & C. butyricum & C. butyricum & $\mathrm{F} 1-\mathrm{b}$ \\
\hline & & & C. sporogens & $\begin{array}{l}\text { C. sporogens/ } \\
\text { C. botulinum }\end{array}$ & C. sporogens & F1-s \\
\hline & F4 & $\begin{array}{l}\text { Swab of inner wall of breast } \\
\text { milk bag }\end{array}$ & C. sporogens & $\begin{array}{l}\text { C. sporogens/ } \\
\text { C. botulinum }\end{array}$ & C. sporogens & F4-s \\
\hline & F5 & $\begin{array}{l}\text { Swab of inner wall of NEC-infant } \\
\text { incubator }\end{array}$ & C. butyricum & C. butyricum & C. butyricum & $\mathrm{F} 5-\mathrm{b}$ \\
\hline & \multirow[t]{2}{*}{ F6 } & \multirow{2}{*}{$\begin{array}{l}\text { Swab of handle of NEC-infant } \\
\text { incubator }\end{array}$} & C. butyricum & C. butyricum & C. butyricum & F6-b \\
\hline & & & C. sporogens & $\begin{array}{l}\text { C. sporogens/ } \\
\text { C. botulinum }\end{array}$ & C. sporogens & F6-s \\
\hline \multirow[t]{3}{*}{ Samples associated with case B } & \multirow[t]{2}{*}{ F7 } & \multirow[t]{2}{*}{ Stool } & C. butyricum & C. butyricum & C. butyricum & F7-b \\
\hline & & & C. sporogens & $\begin{array}{l}\text { C. sporogens/ } \\
\text { C. botulinum }\end{array}$ & C. sporogens & F7-s \\
\hline & F8 & Powdered infant formula & C. butyricum & C. butyricum & C. butyricum & F8-b \\
\hline $\begin{array}{l}\text { Samples associated with } \\
\text { non-NEC infant }\end{array}$ & F36 & Breast milk & C. sporogens & $\begin{array}{l}\text { C. sporogens/ } \\
\text { C.botulinum }\end{array}$ & C. sporogens & F36-s \\
\hline \multirow{6}{*}{$\begin{array}{l}\text { Swabs of the medical } \\
\text { equipment in the ward }\end{array}$} & \multirow[t]{2}{*}{ F13 } & \multirow[t]{2}{*}{ Inner wall of refrigerator } & C. butyricum & C. butyricum & C. butyricum & F13-b \\
\hline & & & C. sporogens & $\begin{array}{l}\text { C. sporogens/ } \\
\text { C. botulinum }\end{array}$ & C. sporogens & F13-s \\
\hline & $\mathrm{F} 16$ & ECG monitor & C. butyricum & C. butyricum & C. butyricum & F16-b \\
\hline & $\mathrm{F} 17$ & Infusion pump & C. butyricum & C. butyricum & C. butyricum & F17-b \\
\hline & $\mathrm{F} 18$ & Stethoscope & C. butyricum & C. butyricum & C. butyricum & F18-b \\
\hline & F19 & Public phone & C. butyricum & C. butyricum & C. butyricum & F19-b \\
\hline \multirow{4}{*}{$\begin{array}{l}\text { Hand swabs of medical } \\
\text { staffs }\end{array}$} & $\mathrm{F} 21$ & Staff 1 & C. butyricum & C. butyricum & C. butyricum & F21-b \\
\hline & $\mathrm{F} 22$ & Staff 2 & C. butyricum & C. butyricum & C. butyricum & $\mathrm{F} 22-\mathrm{b}$ \\
\hline & $\mathrm{F} 23$ & Staff 3 & C. butyricum & C. butyricum & C. butyricum & F23-b \\
\hline & $\mathrm{F} 27$ & Staff 7 & C. butyricum & C. butyricum & C. butyricum & F27-b \\
\hline \multirow{2}{*}{$\begin{array}{l}\text { Swabs of articles for } \\
\text { neonate daily use }\end{array}$} & \multirow[t]{2}{*}{ F29 } & \multirow{2}{*}{$\begin{array}{l}\text { Inner wall of a box for holding } \\
\text { breast milk bag }\end{array}$} & C. butyricum & C. butyricum & C. butyricum & F29-b \\
\hline & & & C. sporogens & $\begin{array}{l}\text { C. sporogens/ } \\
\text { C. botulinum }\end{array}$ & C. sporogens & F29-s \\
\hline
\end{tabular}

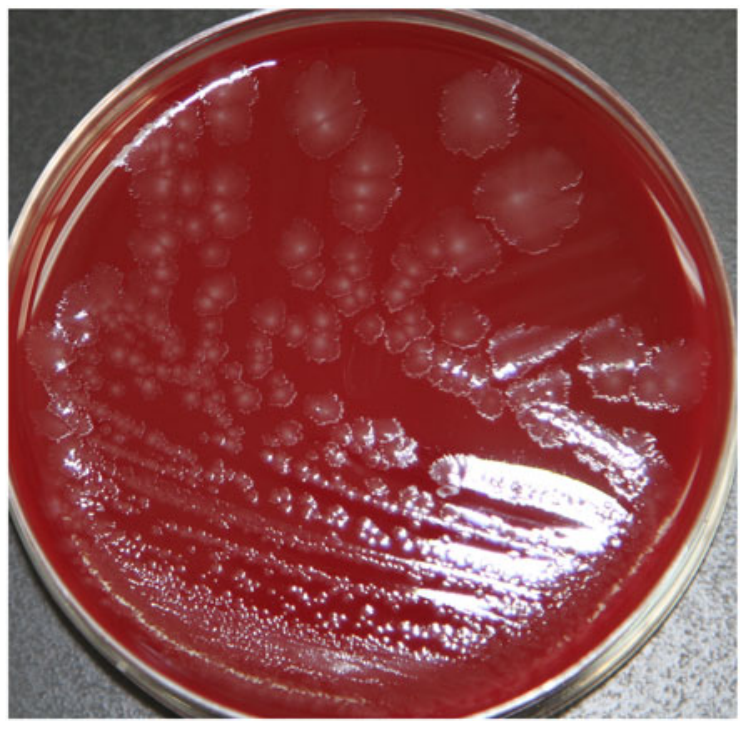

Fig. 1. Morphology on Columbia blood agar of C. butyricum.
Normally, in the NICU in this hospital, only the floor and desk surfaces are disinfected once daily with sodium trichloroisocyanurate at a concentration of $500 \mathrm{mg} / \mathrm{L}$. After this outbreak, based on our laboratory results, the hospital implemented control measures including thorough daily disinfection of the wards, hands of the medical staff, all articles for neonate daily use, and infant feeding supplies. In total, 24 swab samples were subsequently taken from the ward environment, the hands of the attending medical staff, and disposable items for neonate daily use after interventions (Table 1). All were negative for Clostridium. No NEC cases were recorded after disinfection. Therefore, the outbreak of NEC was suspected to be correlated with the contamination of Clostridium, especially C. butyricum.

\section{PFGE-based pulsotyping}

All 15 isolates of $C$. butyricum cultured in this investigation were subjected to pulsotyping, and a single profile was recorded (Fig. 2). This finding suggests that all C. butyricum isolates shared the same pulsotype, and, furthermore, that both PIF intended for infant 


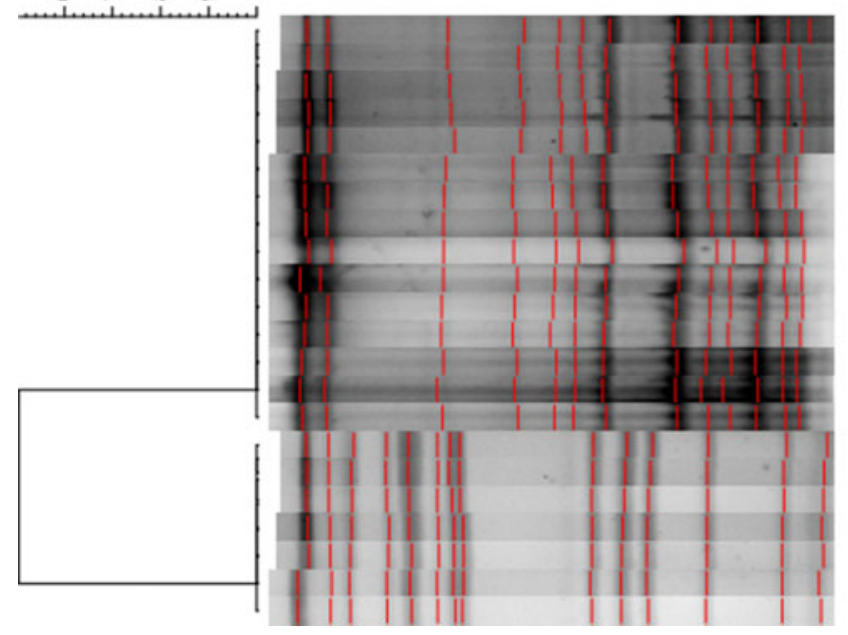

\begin{tabular}{|c|c|}
\hline strain number & strain name \\
\hline$F S-b$ & C.butyricum \\
\hline$F 1-b$ & C.butyricum \\
\hline$F 6-b$ & C.butyricum \\
\hline F 13-b & C.butyricum \\
\hline$F 29-0$ & C.butyricum \\
\hline$F 21-b$ & C.butyricum \\
\hline$F 22=0$ & C.butyricum \\
\hline F23-b & C.butyricum \\
\hline$F 27-b$ & C.butyricum \\
\hline F $16-b$ & C.butyricum \\
\hline$F 17-b$ & C.butyricum \\
\hline F18-b & C.butyricum \\
\hline F19-0 & C.butyricum \\
\hline$F 8-0$ & C.butyricum \\
\hline$F 7 \cdot b$ & C.butyricum \\
\hline FG-s & C.sporogenes \\
\hline F 1 -s & C.sporogenes \\
\hline F4-s & C.sporogenes \\
\hline F13-s & C.sporogenes \\
\hline F2O-s & C.sporogenes \\
\hline F36-s & C.sporogenes \\
\hline F7.s & C.sporogenes \\
\hline
\end{tabular}

Fig. 2. Pulsotypes of 15 C. butyricum and 7 C. sporogenes isolates cultured from samples with Smal restrictive endonuclease. consumption and the ward environment were contaminated with the same strain of C. butyricum. According to the PFGE analysis, the 7 C. sporogenes isolated from this investigation shared the same profile (Fig. 2). Thus, the C. sporogenes isolated from the infant's intestine may have been acquired from the contaminated breast milk.

\section{Whole-genome sequencing of two representative isolates of C. butyricum}

Two complete genomes were determined by PacBio RSII long-read sequencing chemistries to an average coverage of $163 \times$ for the F1-b and $137 \times$ for F5-b. The genome sequence of 2 C. butyricum isolates consisted of a single circular chromosome and 1 circular megaplasmid. The chromosome of $C$. butyricum F1-b consisted of 3,864,433 bp $(3,864,393$ bp for F5-b) with 3,472 (3,469 for F5-b) coding sequences. The average guanine-cytosine (GC) content was $28.88 \%$ for both genomes. The total size of the 1 plasmid from isolate F1-b was 882,416 bp (882,389 for F5-b), with an average GC content of $28.51 \%$ for both. For strain comparison, a reference strain C. butyricum KNU-L09 from NCBI was obtained (Supplementary Table S1 online). To identify potential virulence-encoding genes in the 2 strains of $C$. butyricum, these genomes were used to query those factors listed in the VFDB. No virulence genes were identified on the plasmids (Supplementary Fig. S1 online). The nanH gene was found in the chromosome of 2 isolates but was not present in the reference strain (Fig. 3). In particular, the nanH gene was located in a transposon: IS1182, IS66, IS200/605, IS91, IS256, IS3, and IS30 were flanking it (Fig. 4). Virulence factors, including hemolysin A, hemolysin B, hemolysin C, and hemolysin III family protein identified previously by Cassir et $\mathrm{al}^{8}{ }^{8}$ enterotoxin identified by Kwok et $\mathrm{al}^{22}$ were detected in both C. butyricum strains sequenced in this investigation along with the reference strain (Fig. 3 and Table 3).

A phylogenetic tree based on the core genome alignment was constructed to analyze the evolutionary relationships between our 2 isolates and 16 C. butyricum strains from NCBI (Supplementary Fig. S2 online). The tree revealed that the 2 C. butyricum isolates were in the same clade, a finding consistent with the PFGE result.

\section{Discussion}

Outbreak of NEC in neonates, especially in preterm neonates caused by C. butyricum is known, but globally, only a few have been reported. This outbreak of NEC occurred in a neonatal ward with evidence of association between the presence of toxic C. butyricum strains recovered in the stool samples taken from neonates and the occurrence of NEC. These results are in line with earlier reports. ${ }^{13}$ Those C. butyricum cultured from different environmental and stool samples during outbreak shared the same pulsotype, suggesting a common origin. To the best of our knowledge, this is the first report describing a NEC outbreak linked to the infection of C. butyricum in China.

Notably, some medical instruments, disposable items for neonate daily use, neonate food, and incubators that came into contact with the medical staff were all C. butyricum positive. All C. butyricum from these samples shared the same pulsotype with those isolates recovered from both swabs taken from the hands of the medical staff and NEC patient stools. Data matching led us to strengthen the involvement of C. butyricum in the occurrence of NEC independent of intrinsic factors. In particular, facilities and items in the ward that the medical staff touched were all contaminated with $C$. butyricum. These findings demonstrate that the medical staff themselves may have contributed to transmission of C. butyricum from the original source of contamination to these NEC cases. Additionally, breast milk, the inner wall of breast milk bags, as well as a box for holding breast milk bags were contaminated by $C$. sporogenes with indistinguishable PFGE profile. Thus, the contamination may have come from the breast milk due to mother's poor hygiene habits or from breast milk collection equipment or materials. Although concurrent contamination of C. butyricum and C. sporogenes in some samples was detected, no literature-based evidence has linked NEC outbreaks with infections associated with C. sporogenes. On the other hand, no NEC cases were reported after the ward disinfection following the outbreak, and all samples collected were negative for Clostridium. It is tempting to speculate that the outbreak of NEC reported in the present study was associated with the nosocomial infection of C. butyricum. 


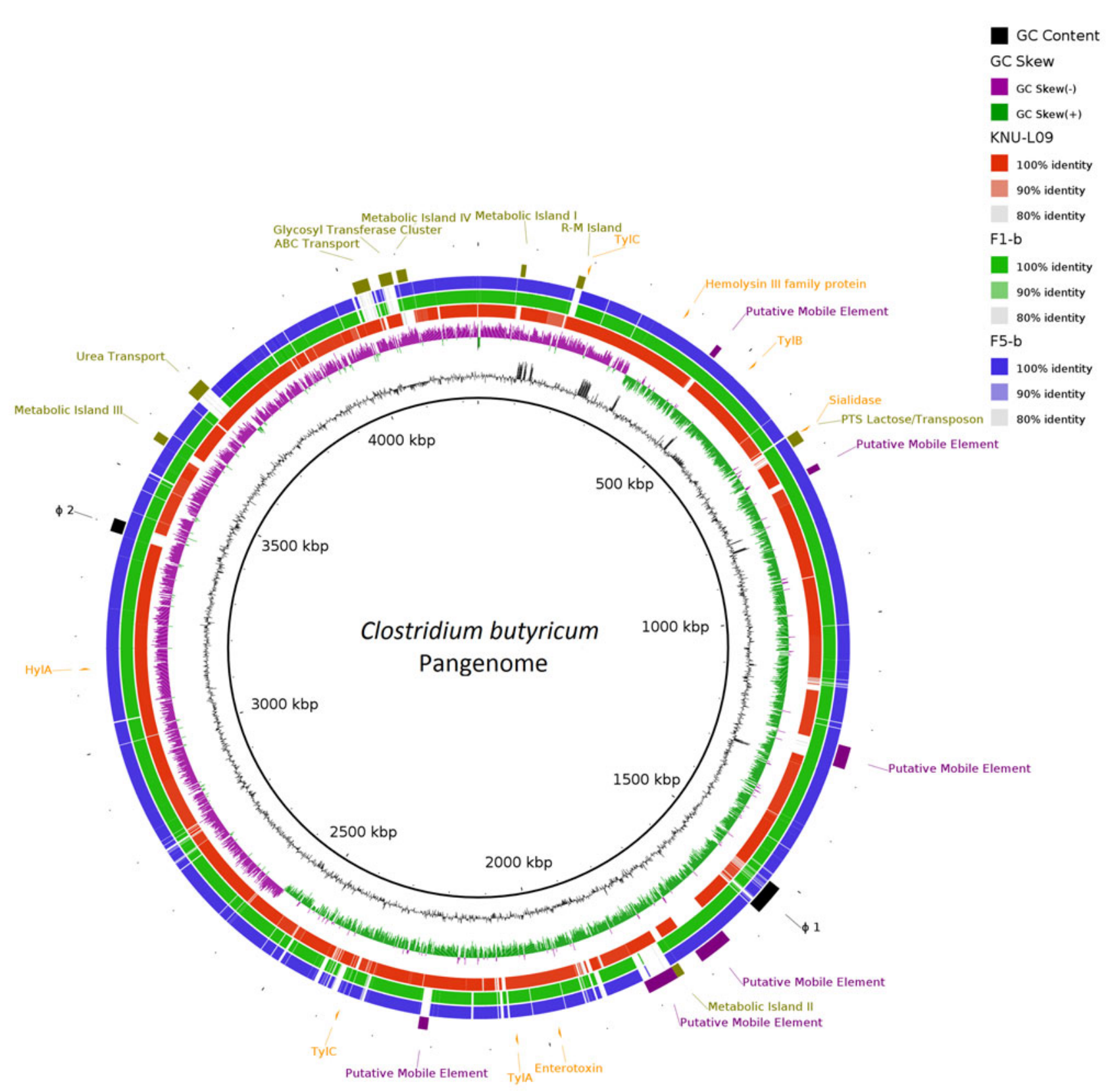

Fig. 3. Major virulence genes of $C$. butyricum F1-b and F1-5 compared with reference strain C. butyricum KNU-L09 (accession no. NZ_CP013252). Beginning from the inside out represents the following features: GC content (black); GC skew (green and purple); C. butyricum KNU-L09 genome (red); C. butyricum F1-b genome (green); C. butyricum F5-b genome (blue); transport genes (shown in green font), putative mobile element (shown in purple font), phage genes (shown in black font) and virulence factor genes (shown in orange font).

NEC-like lesions have been found in animal models when C. butyricum cultures were administered orally. ${ }^{9-12}$ This may be due to the production of butyric acid by the bacterium, whose effect on the intestinal barrier is paradoxical. Butyrate at a low concentration $(2 \mathrm{mM})$ promotes intestinal barrier function but significantly reduces it at a high concentration $(8 \mathrm{mM}) .^{9}$ Thus, a working hypothesis to explain the events described herein involves the overproduction of short-chain fatty acids in the bowel, which in turn may play an important role in the pathogenesis of NEC.

Our WGS data indicated that sialidase, hemolysin, and enterotoxin virulence factors were located in the chromosome of both C. butyricum. The sialidase encoding gene was unique to these 2 isolates. Increasing evidence indicates that sialidase and sialic acid play significant roles in growth and colonization of the intestines by bacterial pathogens, such as C. perfringens and others. ${ }^{23-29}$ These factors can help the pathogen avoid the host immune system defense. $^{30}$ The sialidase encoding gene nanH identified in this study has not been previously reported in C. butyricum. ${ }^{31,32}$ The translated protein product of this gene shares $75.853 \%$ identity with the nan $H$ exo-alpha-sialidase from $C$. perfringens (accession no. ATD49144.1). Among those hemolysins detected in the 2 isolates, the pore-forming $\beta$-hemolysin has been described as the main virulence factor capable of inducing enterocyte necrotic lesions. Purified $\beta$-hemolysin affects the integrity of epithelial 
Table 3. List of Virulence Genes in F1-b and F5-b

\begin{tabular}{|c|c|c|c|c|}
\hline \multirow[b]{2}{*}{ Gene Name } & \multicolumn{2}{|c|}{ Locus Tag } & \multirow[b]{2}{*}{ Protein Name } & \multirow[b]{2}{*}{ Reference } \\
\hline & $\mathrm{F} 1-\mathrm{b}$ & $\mathrm{F} 5-\mathrm{b}$ & & \\
\hline nanH & EBL75_05455 & EBQ27_05455 & Sialidase & VFDB \\
\hline tlyA & EBL75_11590 & EBQ27_11595 & Hemolysin A & Cassir et $\mathrm{al}^{9} 2015$ \\
\hline tlyB & EBL75_04805 & EBQ27_04805 & Hemolysin B & Cassir et $\mathrm{al}^{9} 2015$ \\
\hline tlyc & EBL75_20695 & EBQ27_20700 & Hemolysin C & Cassir et al $^{9} 2015$ \\
\hline$\cdots$ & EBL75_04095 & EBQ27_04095 & Hemolysin III family protein & Cassir et $\mathrm{al}^{9} 2015$ \\
\hline hylA & EBL75_16000 & EBQ27_16000 & $\beta$-Hemolysin & Cassir et $\mathrm{al}^{9} 2015$ \\
\hline$\ldots$ & EBL75_11210 & EBQ27_11215 & Enterotoxin & Kwok et $\mathrm{al}^{23} 2014$ \\
\hline
\end{tabular}

Note: “..." indicates that there was no gene name from Genbank file.

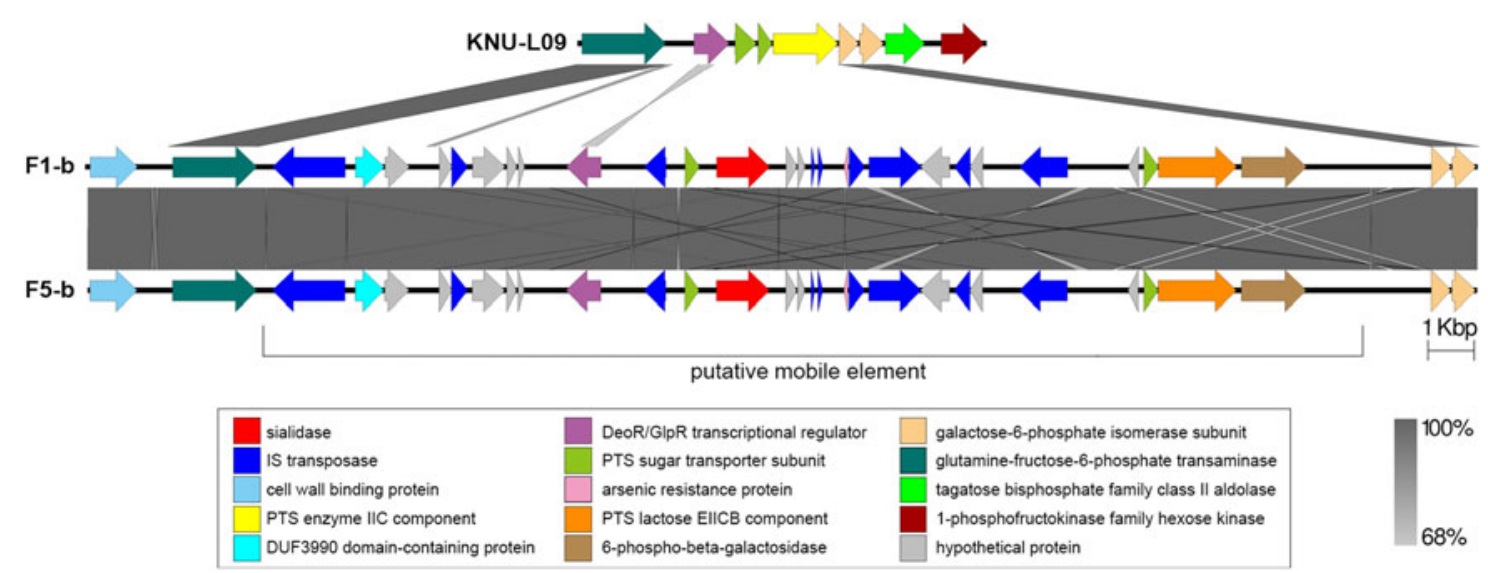

Fig. 4. Genomic environment analysis of phosphotransferase system (PTS) lactose/transposon element which were extracted from whole-genome sequencing (WGS)-based analysis. EasyFig version 1.0 software was using to show the BLAST results. The IS transposases from left to right: IS1182, IS66, IS200/605, IS91, IS256, IS3 and IS30. PTS sugar transporter subunit including PTS lactose/cellobiose transporter subunit IIA, PTS fructose transporter subunit IIB and PTS sugar transporter subunit IIA.

cell monolayers and induces colonic epithelial cell damage. ${ }^{33}$ Enterotoxin is likely responsible for the most common and important human gastrointestinal illnesses caused by C. perfringens. ${ }^{34}$ Many studies have reported that enterotoxigenic strains of C. perfringens have previously been related to enteritis in human and several animal species. ${ }^{35-37}$

Although the pathogenic mechanism of NEC elaborated by C. butyricum remains to be fully understood, based on these findings in our study, we hypothesize that the sialidase might promote the growth and colonizing of the intestines with C. butyricum and possibly protect it from the hosts immune system defense. The hemolysin and enterotoxin factors may then act to induce intestinal cell damage. Interestingly, we found the sialidase encoding gene located on a putative mobile genetic element, and this virulence gene might be transferred from other pathogens such as C. perfringens. This observation suggests that a similar mechanism may exist in the case of other clostridial species (ie, C. perfringens, C. neonatale).

Our study has some limitations. First, the stool samples were obtained from only 2 of 15 NEC patients because most patients (11 in total) had recovered fully and had been discharged from the hospital at the time of sampling and because feces samples produced by 2 patients were too small for analysis owing to fasting and water-free treatment. Secondly, only 2 representative strains of 15 C. butyricum cultured from samples linked to NEC cases were selected for WGS because all 15 strains shared the same PFGE profile. The remaining strains will be sequenced later to elucidate the genetic differences among them.

In conclusion, our findings show an association between NEC and C. butyricum contamination within the hospital. Monitoring of this bacterium in the neonatal ward and pediatric outpatient unit as well as good medical practices should be reinforced and strictly implemented. Moreover, a more extensive study to describe transmission routes, and the possible toxigenic mechanism of C. butyricum involved in the pathogenesis of NEC warrants further investigation.

Supplementary material. To view supplementary material for this article, please visit https://doi.org/10.1017/ice.2019.289.

Acknowledgments. We thank Fang He and Yanfang Geng, the staffs of hospital for their support of this study.

Financial support. This study was supported by research grants from National Natural Science Foundation of China (contract no. 81673176) and Beijing Natural Science Foundation (contract no. 7172165).

Conflicts of interest. All authors report no conflicts of interest relevant to this article. 


\section{References}

1. Hack M, Horbar JD, Malloy MH, et al. Very low birth weight outcomes of the National Institute of Child Health and Human Development Neonatal Network. Pediatrics 1991;87:587-597.

2. Uauy RD, Fanaroff AA, Korones SB, et al. Necrotizing enterocolitis in very low birth weight infants: biodemographic and clinical correlates. National Institute of Child Health and Human Development Neonatal Research Network. J Pediatr 1991;119:630-638.

3. Snyder CL, Gittes GK, Murphy JP, et al. Survival after necrotizing enterocolitis in infants weighing less than 1,000 g: 25 years' experience at a single institution. J Pediatr Surg 1997;32:434-437.

4. Claud EC, Walker WA. Hypothesis: inappropriate colonization of the premature intestine can cause neonatal necrotizing enterocolitis. FASEB J 2001;15:1398-1403.

5. Kathleen Sim, Alexander G. Shaw, Paul Randell, et al. Dysbiosis anticipating necrotizing enterocolitis in very premature infants. Clin Infect Dis 2015;60: 389-397.

6. Waligora-Dupriet A-J, Dugay A, Auzeil N, et al. Evidence for clostridial implication in necrotizing enterocolitis through bacterial fermentation in a gnotobiotic quail model. Pediatr Res 2005;58:629-635.

7. Sturm R, Staneck JL, Stauffer LR, et al. Neonatal necrotizing enterocolitis associated with penicillin-resistant, toxigenic Clostridium butyricum. Pediatrics 1980;66:928-931.

8. Cassir N, Benamar S, Khalil JB, et al. Clostridium butyricum strains and dysbiosis linked to necrotizing enterocolitis in preterm neonates. Clin Infect Dis 2015;61:1107-1115.

9. Peng L, He Z, Chen W, et al. Effects of butyrate on intestinal barrier function in a Caco-2 cell monolayer model of intestinal barrier. Pediatr Res 2007;61: 37-41.

10. Azcarate-Peril MA, Foster DM, Cadenas MB, et al. Acute necrotizing enterocolitis of preterm piglets is characterized by dysbiosis of ileal mucosa-associated bacteria. Gut Microbes 2011;2:-234-243.

11. Popoff MR, Szylit O, Ravisse P, et al. Experimental cecitis in gnotoxenic chickens monoassociated with Clostridium butyricum strains isolated from patients with neonatal necrotizing enterocolitis. Infect Immun 1985;47: 697-703.

12. Waligora-Dupriet AJ, Dugay A, Auzeil N, et al. Short-chain fatty acids and polyamines in the pathogenesis of necrotizing enterocolitis: kinetics aspects in gnotobiotic quails. Anaerobe 2009;15:138-144.

13. Hyatt DR, Joens LA. Analysis of the lytic activity of the Serpulina hyodysenteriae hemolysin. Infect Immun 1997;65:4877-4879.

14. US Food and Drug Administration. Chapter 17, Clostridium botulinum. In Bacteriological Analytical Manual. Washington, DC: FDA; 2001.

15. Dong YP, Li ZG, Li FQ. Comparison of mouse assay and pulsed field gel electrophoresis of Clostridium sporogenes isolated from China and New Zealand [in Chinese]. Chin J Food Hygiene 2016;28:40-43.

16. Jandrasits C, Dabrowski PW, Fuchs S, et al. seq-seq-pan: building a computational pan-genome data structure on whole genome alignment. $B M C$ Genomics 2018;19:47.

17. Alikhan NF, Petty NK, Ben Zakour NL, et al. BLAST ring image generator (BRIG): simple prokaryote genome comparisons. BMC Genomics 2011;12:402.

18. Arndt D, Grant JR, Marcu A, et al. PHASTER: a better, faster version of the PHAST phage search tool. Nucleic Acids Res 2016;44:W16-W21.
19. Kearse M, Moir R, Wilson A, et al. Geneious Basic: an integrated and extendable desktop software platform for the organization and analysis of sequence data. Bioinformatics 2012;28:1647-1649.

20. Treangen TJ, Ondov BD, Koren S, et al. The Harvest suite for rapid coregenome alignment and visualization of thousands of intraspecific microbial genomes. Genome Biol 2014;15:524.

21. He Z, Zhang H, Gao S, et al. Evolview v2: an online visualization and management tool for customized and annotated phylogenetic trees. Nucleic Acids Res 2016;44:W236-W241.

22. Kwok JS, Ip M, Chan TF, et al. Draft genome sequence of Clostridium butyricum strain NOR 33234, isolated from an elderly patient with diarrhea. Genome Announc 2014 Dec 24;2(6): pii: e01356-14. doi: 10.1128/genomeA. 01356-14.

23. Lewis AL, Lewis WG. Host sialoglycans and bacterial sialidases: a mucosal perspective. Cell Microbiol 2012;14:1174-1182.

24. Li J, Sayeed S, Robertson S, et al. Sialidases affect the host cell adherence and epsilon toxin-induced cytotoxicity of Clostridium perfringens type D strain CN3718. PLoS Pathog 2011 Dec;7(12):e1002429. doi: 10.1371/journal.ppat. 1002429.

25. Almagro-Moreno S, Boyd EF. Sialic acid catabolism confers a competitive advantage to pathogenic Vibrio cholerae in the mouse intestine. Infect Immun 2009;77:3807-3816.

26. Brittan JL, Buckeridge TJ, Finn A, et al. Pneumococcal neuraminidase A: an essential upper airway colonization factor for Streptococcus pneumoniae. Mol Oral Microbiol 2012;27:270-283.

27. Awad MM, Singleton J, Lyras D. The sialidase NanS enhances non-TcsL mediated cytotoxicity of Clostridium sordellii. Toxins (Basel) 2016;8(6): pii: E189. doi: 10.3390/toxins8060189.

28. Chang DE, Smalley DJ, Tucker DL, et al. Carbon nutrition of Escherichia coli in the mouse intestine. Proc Natl Acad Sci U S A 2004;101:7427-7432.

29. Jeong HG, Oh MH, Kim BS, et al. The capability of catabolic utilization of $\mathrm{N}$-acetylneuraminic acid, a sialic acid, is essential for Vibrio vulnificus pathogenesis. Infect Immun 2009;77:3209-3217.

30. Severi E, Hood DW, Thomas GH. Sialic acid utilization by bacterial pathogens. Microbiology 2007;153:2817-2822.

31. Li J, Uzal FA, McClane BA. Clostridium perfringens sialidases: potential contributors to intestinal pathogenesis and therapeutic targets. Toxins (Basel) 2016;8(11): pii: E341. doi: 10.3390/toxins8110341.

32. Li J, McClane BA. The sialidases of Clostridium perfringens type D strain CN3718 differ in their properties and sensitivities to inhibitors. Appl Environ Microbiol 2014;80:1701-1709.

33. Hsu T, Hutto DL, Minion FC, et al. Cloning of a beta-hemolysin gene of Brachyspira (Serpulina) hyodysenteriae and its expression in Escherichia coli. Infect Immun 2001;69:706-711.

34. Miyakawa ME, Saputo J, Leger JS, et al. Necrotizing enterocolitis and death in a goat kid associated with enterotoxin (CPE)-producing Clostridium perfringens type A. Can Vet J 2007;48:1266-1269.

35. Bos J, Smithee L, McClane B, et al. Fatal necrotizing colitis following a foodborne outbreak of enterotoxigenic Clostridium perfringens type A infection. Clin Infect Dis 2005;40(10):e78-e83. doi: 10.1086/429829.

36. Songer JG. Clostridial enteric diseases of domestic animals. Clin Microbiol Rev 1996;9:216-234.

37. Dray T. Clostridium perfringens type A and beta2 toxin associated with enterotoxemia in a 5-week-old goat. Can Vet J 2004;45:251-253. 